Commun. math. Phys. 6, 312-342 (1967)

\title{
Complete Sets of Solutions of Linear Lorentz Covariant Field Equations with an Infinite Number of Field Components
}

\author{
W. RüHL* \\ The Rockefeller University \\ New York \\ Received April 15, 1967
}

Abstract. We study field equations of the Gelfand-Yaglom type

$$
\left(-i \Gamma_{\mu} \frac{\partial}{\partial x_{\mu}}-x\right) \Psi(x)=0
$$

where $\Psi$ transforms as a unitary representation of the inhomogeneous Lorentz group. We construct a complete set of solutions of this equation. This set includes solutions with spacelike momentum. Our method makes use of the decomposition of unitary representations of the homogeneous Lorentz group into unitary representations of the little groups $S U(2)$ and $S U(1,1)$. The covariant operators $\Gamma_{\mu}$ are written as differential operators on homogeneous spaces. For some classes of equations we calculate the mass spectrum explicitly.

\section{Introduction}

We study equations of the type

$$
\left(-i \Gamma_{\mu} \frac{\partial}{\partial x_{\mu}}-x\right) \Psi(x)=0
$$

where $x$ is a real parameter different from zero, and $\Psi$ transforms as a finite direct sum of unitary irreducible representations of the homogeneous Lorentz group. This means that for any element " $a$ " of the homogeneous Lorentz group $S L(2, C)$, we have a transformation

$$
\begin{gathered}
\Psi(x) \underset{a}{\longrightarrow} \Psi^{\prime}(x), \\
\Psi^{\prime}(x)=U_{a} \Psi\left(\Lambda_{a}^{-1} x\right)
\end{gathered}
$$

such that $\Psi$ is a vector in a representation space of $S L(2, C)$ depending on the argument $x$ and $U_{a}$ is a unitary operator in this space representing the element " $a$ ". Further we define

$$
\left(\Lambda_{a} x\right)_{\mu}=\Lambda_{a \mu}^{\nu} x_{\nu} .
$$

* Permanent address: CERN, Geneva. 
Covariance of the equation can be achieved by the requirement

$$
U_{a}^{-1} \Gamma_{\mu} U_{a}=\Lambda_{a \mu}^{\nu} \Gamma_{v} .
$$

We assume that the operators $\Gamma_{\mu}$ possess a common invariant dense domain in the representation space.

In the momentum representation

$$
\Psi(x)=(2 \pi)^{-2} \int e^{i p x} \Psi(p) d^{4} p
$$

we can write the equation as

$$
\left(p^{\mu} I_{\mu}-\varkappa\right) \Psi(p)=0 .
$$

The four momentum $p_{\mu}$ appearing in this equation may a priori be timelike,

spacelike,

$$
\left(p^{0}\right)^{2}-(\mathbf{p})^{2}=\mu^{2}>0
$$

or lightlike,

$$
\left(p^{0}\right)^{2}-(\mathbf{p})^{2}=-\lambda^{2}, \quad \lambda>0
$$

$$
\left(p^{0}\right)^{2}-(\mathbf{p})^{2}=0
$$

Special examples of such field equations have first been investigated by MaJorana [1]. The general theory of Lorentz covariant equations has been developed by GELFAND and YAGLOM in a series of papers [2] ${ }^{1}$. These authors study in particular timelike solutions and neglect the spacelike solutions, which has been pointed out by BARGManN [3]. The aim of this article is to discuss spacelike and timelike representations simultaneously and display a general framework which enables us to obtain a complete set of solutions.

To illustrate what we mean by "complete set" in this context, let me recall what the Plancherel theorem for a Lie group says. It asserts the completeness and orthogonality of all matrix elements of a certain subset of unitary irreducible representations of the group in the Hilbert space of square integrable functions on the group. In addition, the theorem defines a measure in this subset of representations, which tells us how to sum up (in the discrete or continuous sense) the contributions of different representations. This is the Plancherel measure.

The Plancherel theorem for the inhomogeneous Lorentz group has been established recently [4]. Any solution $\Psi(x)$ represents a certain function on the inhomogeneous Lorentz group. If it is sufficiently regular it can be decomposed into components belonging to irreducible unitary representations of the inhomogeneous Lorentz group relying on the completeness and orthogonality of the functions specified in the Plancherel theorem. These components have definite mass and spin. It turns out

${ }^{1}$ A short review of the results can be found in Ref. [3], an exhaustive discussion in the textbook, Ref [5].

22 Commun. math. Phys., Vol. 6 
that in the case when the unitary representation $U_{a}$ of $S L(2, C)$ is a finite direct sum of irreducible representations, a finite number of functions

$$
M^{2}=M^{2}(J)
$$

exists which relate the mass $M$ and the spin $J$ of the components appearing in a solution. The parts $M^{2}>0$ of these functions are known since the work of Gelfand and Yaglom [2]. Our solutions cover also the domain of negative values $M^{2}$.

We do not want to study here which regularity properties a solution must possess to be decomposable and how this decomposition converges. Consequently we regard the definition of a "complete set of solutions" only as a technical term: We consider all plane wave solutions

$$
\Psi_{p}(x)=e^{i p x} \Psi(p)
$$

such that $\Psi$ belongs to any irreducible representation of the little group corresponding to $p$ which is contained in the reduction of the unitary representation of the group $S L(2, C)$.

In practice we proceed as follows. If the momentum is timelike we may bring the equation to the form

$$
\left(\Gamma_{0} \mu-\varkappa\right) \Psi(\mu)=0, \quad \mu \lessgtr 0 .
$$

We solve this eigenvalue equation for $\Gamma_{0}$ which yields an eigenvector $\Psi(\mu)$ for any point $\mu$ of the spectrum of the self-adjoint operator $x \Gamma_{0}^{-1}$. The spectrum of this operator is discrete, the eigenvectors are normalizable. Any point $p$ on the timelike orbit

$$
\left\{p:\left(p^{0}\right)^{2}-(\mathbf{p})^{2}=\mu^{2}, \operatorname{sign} p^{0}=\operatorname{sign} \mu\right\}
$$

can be reached from the rest system by a pure Lorentz transformation $a(p) \in S L(2, C)$,

We may define $\Psi(p)$ by

$$
a(p) \cdot p \cdot a(p)^{\dagger}=\mu .
$$

$$
\Psi(p)=U_{a(p)} \Psi(\mu) .
$$

To find the spectrum of $\Gamma_{0}$ we use the fact that $\Gamma_{0}$ commutes with $U_{u}$, where $u$ is any element of the timelike little group $S U(2)$. Schur's lemma implies that $\Gamma_{0}$ reduces to blocks. If $\Psi$ is decomposed in components $\Psi_{j}$,

$$
\begin{aligned}
\Psi & =\sum_{j} \oplus \Psi_{j}, \\
\Psi_{j} & =P_{j} \Psi,
\end{aligned}
$$

where $P_{j}$ is the projection operator on the subspace which carries all representations of $S U(2)$ to the spin $j$, we may express Schur's lemma as

$$
\Gamma_{0}=\sum_{j} \oplus P_{j} \Gamma_{0} P_{j} \equiv \sum_{j} \oplus\left(\Gamma_{0}\right)_{j} .
$$


The projection $P_{j}$ is a well known operation [5]. The Peter and Weyl theorem [6] for the group $S U(2)$, which is the version of the Plancherel theorem for bicompact groups, states explicitly how to construct this operator.

We can proceed similarly in the case of spacelike solutions. We can reduce the field equation to

$$
\left(\Gamma_{3} \lambda-x\right) \Psi(\lambda)=0
$$

such that $\lambda$ appears as the eigenvalue of the operator $x \Gamma_{3}^{-1}$, which is again self-adjoint. The spectrum of this operator contains always a continuum with corresponding non-normalizable eigenvectors. To find the spectrum of $\Gamma_{3}$ we must decompose the unitary representations of $S L(2, C)$ into irreducible unitary representations of $S U(1,1)$. We do this for the principal series of $S L(2, C)$ with the help of the Plancherel theorem for the group $S U(1,1)[7,8]$.

We note that the mass square operator $M^{2}$ can be defined by

$$
M^{2}= \begin{cases}+x^{2} \cdot \Gamma_{0}^{-2}, & \text { in the timelike, } \\ -x^{2} \cdot \Gamma_{3}^{-2}, & \text { in the spacelike case } .\end{cases}
$$

Finally there are the solutions on the lightcone. In principle these solutions present no further difficulties. The techniques used to find the timelike and spacelike solutions can be generalized to yield also the solutions on the lightcone. Since zero appears as an accumulation point of both the positive and negative part of the $M^{2}$-spectrum (see Section V), the solutions on the lightcone are of no particular interest. Therefore we do not go into the detailed construction of these solutions but state their main properties:

$\alpha$ ) the point zero has the same multiplicity as the spacelike part of the $M^{2}$-spectrum just below zero,

$\beta$ ) half of the solutions lie on the future, the other on the past halfcone,

$\gamma)$ all solutions belong to representations with continuous spin, their spins are equal.

The latter property is a consequence of the fact that the Plancherel theorem for the little group $U(1) \times T_{2}$ involves only representations with "continuous spin". To illustrate our assertions we construct the solutions on the lightcone for a simple example of a field equation (Section V, subsection 2).

We present the material as follows. In section II we quote fundamental results of GELFAND and NATMARK on the unitary representations of $S L(2, C)$ [5]. The decomposition into irreducible representations of $S U(2)$ establishes the so-called canonical basis in the space of the $S L(2, C)$ representation. Finally we mention some results of GELFAND and YAGLOM [2]. 
In section III we express the covariant operators $\Gamma_{\mu}$ as differential operators on homogeneous spaces. Applying these operators to the elements of the canonical basis, we find the matrices $\left(\Gamma_{0}\right)_{j}$, which yields some of GELFAND's and YAGLOM's results for the positive part of the $M^{2}$-spectrum.

In section IV we decompose unitary representations of the principal series of $S L(2, C)$ into unitary irreducible representations of $S U(1,1)$. This yields a new basis, the canonical pseudo-basis (some basis vectors are non-normalizable).

In section $V$ we apply the differential operators of section III to the pseudo-basis and complete the mass spectrum.

Our treatment of the matrices $\Gamma_{\mu}$ as differential operators uses unpublished material from Dr. J. Wess. After the completion of this work we learned of a recent publication [9] in which the decomposition of unitary representations of $S U(1,1)$ is achieved with the same method which we develop in Section IV.

\section{Unitary Representations of $S L(2, C)$ [5]}

\section{Functions on Homogeneous Spaces}

In this section we present standard methods and results which enable us to simplify and shorten the discussion of section IV.

We consider the group $K$ of triangular matrices $k$ of the form

$$
k=\left(\begin{array}{cc}
\lambda^{-1} & \mu \\
0 & \lambda
\end{array}\right)
$$

where $\lambda, \mu$ are complex numbers. Points in the homogeneous space

$$
S L(2, C) / K
$$

can be characterized in different fashions; in general we may use any element of $S L(2, C)$ in the rest class which represents the point considered. One way to do this is choosing triangular matrices $z$ of the form

$$
z=\left(\begin{array}{ll}
1 & 0 \\
z & 1
\end{array}\right), \quad z \text { complex }
$$

This is possible only for almost all points of $S L(2, C) / K$, because any element " $a$ " of $S L(2, C)$ can be written

only if $a_{22} \neq 0$.

$$
a=k \cdot z
$$

Unitary matrices $u$,

$$
u=\left(\begin{array}{ll}
u_{11} & u_{12} \\
u_{21} & u_{22}
\end{array}\right)
$$

are another possibility. However, in the decomposition

$$
a=k \cdot u
$$


we may replace $u$ by $\gamma \cdot u$ where $\gamma$ is diagonal and unitary

$$
\gamma=\left(\begin{array}{cc}
e^{i \omega} & 0 \\
0 & e^{-i \omega}
\end{array}\right)
$$

The element $u$ is therefore not unique. A third possibility will be introduced in Section IV.

Unitary representations of $S L(2, C)$ can be realized in Hilbert spaces of functions over $S L(2, C) / K$. For simplicity we consider only the principal series, the supplementary series can be handled similarly [5]. In the Hilbert space $\mathscr{L}^{2}(Z)$ of square integrable functions $f(z)$,

$$
\int|f(z)|^{2} d z<\infty, \quad z=x+i y, \quad d z=d x d y
$$

we define the representation $U_{a}$ as

with

$$
U_{a} f(z)=\alpha(z a) f\left(z^{\prime}\right)
$$

and

$$
\alpha(\alpha)=\left|a_{22}\right|^{m+i \varrho-z} a_{22}^{-m}
$$

$$
z a=k \cdot z^{\prime}, \quad z^{\prime}=\frac{a_{11} z+a_{21}}{a_{12} z+a_{22}} .
$$

Any pair $(m, \varrho)$ where $m$ is integer and $\varrho$ is real defines a unitary irreducible representation $U_{a}$ of the principal series. We call it $S(m, \varrho)$. Two representations $S(m, \varrho), S\left(m^{\prime}, \varrho^{\prime}\right)$ are equivalent if and only if

$$
m^{\prime}=m, \varrho^{\prime}=\varrho \text { or } m^{\prime}=-m, \varrho^{\prime}=-\varrho .
$$

Instead of these parameters we shall make use of another pair:

$$
\begin{aligned}
j_{0} & =\left|\frac{m}{2}\right|, \\
c & =+i \cdot \frac{\varrho}{2} \operatorname{sign} m,
\end{aligned}
$$

which characterizes a representation uniquely. We use the further notation

$$
\sigma=\operatorname{sign} m \text {. }
$$

Let us introduce functions $\varphi(u)$ satisfying the subsidiary condition

$$
\varphi(\gamma u)=e^{i m \omega} \varphi(u)
$$

where $\gamma$ is diagonal and unitary as defined above. By

$$
\int|\varphi(u)|^{2} d \mu(u)<\infty
$$

we define a Hilbert space $\mathscr{L}^{2}{ }_{m}(U)$. The elements $z$ and $u$ are related by

$$
\left(\begin{array}{ll}
1 & 0 \\
z & 1
\end{array}\right)=\left(\begin{array}{cc}
\lambda^{-1} & \mu \\
0 & \lambda
\end{array}\right)\left(\begin{array}{ll}
u_{11} & u_{12} \\
u_{21} & u_{22}
\end{array}\right)
$$


which yields

$$
\begin{aligned}
& z=u_{21} \cdot u_{22}^{-1}, \\
& \lambda=u_{22}^{-1}=e^{i \omega}\left(1+|z|^{2}\right)^{\frac{1}{2}},
\end{aligned}
$$

with an arbitrary phase $\omega$. We can then map $\mathscr{L}^{2}(Z)$ on $\mathscr{L}_{m}^{2}(U)$ by

$$
\varphi(u)=\pi^{\frac{1}{2}} \alpha(u) \cdot f(z(u))
$$

With the parameterization of $u$ (see appendix, subsection 2 )

we obtain

$$
\begin{aligned}
& u_{11}=t^{\frac{1}{2}} e^{i \theta_{1}}, u_{12}=-(1-t)^{\frac{1}{2}} e^{i \theta_{2}}, \\
& u_{21}=-\bar{u}_{12}, \quad u_{22}=\bar{u}_{11},
\end{aligned}
$$

$$
\begin{aligned}
t & =\left(1+|z|^{2}\right)^{-1}, \\
\theta_{1} & =\omega, \theta_{2}=\omega-\psi, \psi=\arg z, \\
\alpha(u) & =e^{i m \omega}\left(1+|z|^{2}\right)^{-i \frac{\varrho}{2}+1} .
\end{aligned}
$$

The invariant measure on $S U(2)$ is

$$
d \mu(u)=\frac{1}{4 \pi^{2}} d t d \theta_{1} d \theta_{2}=\frac{1}{2 \pi^{2}} \cdot \frac{d z}{\left(1+|z|^{2}\right)^{2}} \cdot d \omega
$$

This implies immediately

$$
\int|\varphi(u)|^{2} d \mu(u)=\int|f(z)|^{2} d z .
$$

For the representation $U_{a}$ in $\mathscr{L}_{m}^{2}(U)$ we find

where

$$
U_{a} \varphi(u)=\frac{\alpha(u a)}{\alpha\left(u^{\prime}\right)} \varphi\left(u^{\prime}\right)
$$

$$
u a=k \cdot u^{\prime}
$$

\section{The Canonical Basis}

In the Hilbert space $\mathscr{L}^{2}(U)$ of square integrable functions $\varphi(u)$ on $S U(2)$ we may use all the matrix elements $D_{q p}^{j}(u)$ of all unitary representations $j=0, \frac{1}{2}, 1, \ldots$ as a basis. This is a special case of the theorem of Peter and Weyl [6]. We use that form of $D_{q p}^{j}(u)$ which is defined in the appendix, subsection 2.

With the "Fourier coefficients"

the series

$$
K_{q p}^{j}(\varphi)=\int \varphi(u) \overline{D_{q p}^{j}(u)} d \mu(u)
$$

$$
\sum_{j=0, \frac{1}{2}, 1, \ldots}^{\infty}(2 j+1) \sum_{q, p=-j}^{+j} K_{q p}^{j}(\varphi) D_{q p}^{j}(u)
$$

converges in the mean against $\varphi(u)$. 
Our space $\mathscr{L}_{m}^{2}(U)$ is a subspace of $\mathscr{L}^{2}(U)$. The subsidiary condition on $\varphi(u)$ yields

$$
\begin{aligned}
K_{q p}^{j}(\varphi) & =\int \varphi(u) \overline{D_{q p}^{j}(u)} d \mu(u) \\
& =\int \varphi(\gamma u) \overline{D_{q p}^{j}(\gamma u)} d \mu(u) \\
& =e^{i \omega(m-2 q)} K_{q p}^{j}(\varphi) .
\end{aligned}
$$

We conclude: either $K_{q p}^{j}=0$ or $q=\frac{m}{2}$. The space $\mathscr{L}_{m}^{2}(U)$ can therefore be spanned by the normalized, orthogonal vectors

The phase $\varkappa_{m, e}^{(j)}$,

$$
\varphi_{p}^{j}(u)=(2 j+1)^{\frac{1}{2}} \chi_{m, \varrho}^{(j)} D_{\frac{m}{2}, p}^{j}(u), \quad-j \leqq p \leqq+j .
$$

$$
x_{m, \varrho}^{(j)}=\prod_{\nu=j_{0}}^{j} \frac{i \varrho-2 v}{\sqrt{\varrho^{2}+4 v^{2}}}
$$

has been introduced to bring the matrix elements of the generators into convenient form (see appendix, subsection 4). The basis-vectors $\varphi_{p}^{j}(u)$ form the canonical basis (this notation goes back to NAIMARK [5]).

It is obvious that the basis vectors belonging to a fixed spin $j$ span a $(2 j+1)$-dimensional subspace of $\mathscr{L}^{2}{ }_{m}(U)$ which carries an irreducible representation of $S U(2)$. We have thus established the following result:

In the decomposition of a unitary representation $S(m, \varrho)$ of $S L(2, C)$ into unitary irreducible representations of $S U(2)$, a spin $j$ appears if and only if

$$
j=j_{0}+\text { non-negative integer }
$$

and it appears just once.

Let us now give the corresponding basis in $\mathscr{L}^{2}(Z)$,

$$
f_{p}^{j}(z)=\pi^{-\frac{1}{2}} \alpha(u)^{-1} \varphi_{p}^{j}(u) .
$$

With the results and definitions of appendix, subsections 1 and 2 we obtain

$$
\begin{aligned}
f_{p}^{j}(z)= & {\left[\frac{2 j+1}{\pi} \cdot \frac{\left(j+\frac{m}{2}\right) !\left(j-\frac{m}{2}\right) !}{(j+p) !(j-p) !}\right]^{\frac{1}{2}} \cdot \varkappa_{m, e}^{(j)} \times } \\
& \times\left(1+|z|^{2}\right)^{i \frac{\varrho}{2}-j-1} I_{\frac{m}{2}, p}^{j}(z,-\bar{z}) .
\end{aligned}
$$

$I_{\frac{m}{2}, p}^{j}$ is the principal function defined in appendix, subsection 1.

\section{The Covariant Matrices $\Gamma_{\mu}$}

The representation $U_{a}$ satisfying

$$
U_{a}^{-1} \Gamma_{\mu} U_{a}=\left(\Lambda_{a}\right)_{\mu}^{\nu} \Gamma_{\nu}
$$


can in general not be chosen irreducible but consists of different irreducible parts, which are coupled by the operators $\Gamma_{\mu}$. GELFANd and YAGLOM [5] were able to derive the fundamental theorem:

Two representations $U_{a}^{(1)}, U_{a}^{(2)}$ can be coupled together by $\Gamma_{\mu}$ if and only if one of the following two conditions is satisfied:

a) $\left|m^{(1)}-m^{(2)}\right|=2, \varrho^{(1)}=\varrho^{(2)}$,

B) $m^{(1)}=m^{(2)}, \varrho^{(1)}-\varrho^{(2)}= \pm 2 i$.

The theorem applies to all unitary and non-unitary representations of $S L(2, C)$. If both representations belong to principal series, $\varrho$ is real and only case $\alpha$ ) applies.

If the equation

$$
\left(-i \Gamma_{\mu} \frac{\partial}{\partial x_{\mu}}-x\right) \Psi(x)=0
$$

is also required to be covariant under parity reflections, this may also result in an enlargement of the representation $U_{a}$ to be used. In general, a parity reflection maps the representation $S(m, \varrho)$ on $S(m,-\varrho)$. If these representations are inequivalent, they must be taken together to allow a parity operation. We shall neglect this sort of complication in the sequel.

\section{Covariant Operators as Differential Operators in $\mathscr{L}^{2}(Z)$}

\section{The Problem}

We study operators $T$, defined on a dense domain in the space of the unitary representation $S\left(m_{1}, \varrho_{1}\right)$ of the principal series, with values in the space of the unitary representation $S\left(m_{2}, \varrho_{2}\right)$ of the principal series. We require that $T$ transforms as a finite-dimensional non-unitary representation of $S L(2, C)$ :

$$
U_{a}^{(2)-1} T(z) U_{a}^{(1)}=\left(a_{12} z+a_{22}\right)^{\lambda}\left(\bar{a}_{12} \bar{z}+\bar{a}_{22}\right)^{\mu} T\left(z^{\prime}\right),
$$

$\lambda, \mu$ are non-negative integers ${ }^{2}, z^{\prime}$ is as usual

$$
z^{\prime}=\frac{a_{11} z+a_{21}}{a_{12} z+a_{22}}
$$

$T(z)$ is a polynomial in $z$ of degree $\lambda$ and in $\bar{z}$ of degree $\mu$.

We are going to show that if

$$
\lambda+\mu \text { is even, }
$$

$T(z)$ is a differential operator of order $\frac{1}{2}(\lambda+\mu)$ acting in the space $\mathscr{L}^{2}(X)$ [x are triangular matrices] which carries both representations $S\left(m_{1}, \varrho_{1}\right)$ and $S\left(m_{2}, \varrho_{2}\right)$. If $\lambda+\mu$ is even, $T(z)$ transforms as a Lorentz

${ }^{2}$ The connection with the familiar spins $\left(j_{1}, j_{2}\right)$ is:

$$
\lambda=2 j_{1}, \quad \mu=2 j_{2} \text {. }
$$


tensor. The operators $\Gamma_{\mu}$ are a special case hereof. In their case

and

$$
\lambda=\mu=1
$$

$$
\Gamma_{\mu}=\frac{1}{2} \operatorname{Tr}\left(\sigma_{\mu} T\right) g_{\mu \mu},
$$

where in terms of the $2 \times 2$ matrix $\Gamma$

$$
\Gamma(z)=+\Gamma_{11}-z \Gamma_{12}-\bar{z} \Gamma_{21}+|z|^{2} \Gamma_{22} .
$$

Another case of particular interest are the generators of $S L(2, C)$ belonging to $\lambda=2, \mu=0$ and $\lambda=0, \mu=2$. Their differential operator form can be found by more direct methods, it is given in appendix, subsection 4 .

\section{The Differential Operators}

In the case $\lambda \geqq \mu$ we get, provided

$$
\begin{gathered}
m_{1}=m, \quad m_{2}=m+2 \mu \\
\varrho_{1}=\varrho_{2}=\varrho \\
T(z)_{m \rightarrow m+2 \mu}=(\bar{x}-\bar{z})^{\mu}(x-z)^{-\frac{1}{2} m+\frac{i}{2} \varrho+\frac{1}{2}(\lambda-\mu)} \times \\
\times\left[-2 i \frac{\partial}{\partial x}\right]^{\frac{1}{2}(\lambda+\mu)}(x-z)^{\frac{1}{2}} \frac{1}{2} m-\frac{i}{2} \varrho+\frac{1}{2}(\lambda+\mu),
\end{gathered}
$$

in the case $\lambda \leqq \mu$ we get, provided

$$
\begin{aligned}
& m_{1}=m+2 \lambda, \quad m_{2}=m, \\
& \varrho_{1}=\varrho_{2}=\varrho \text {, } \\
& T(z)_{m+2 \lambda \rightarrow m}=(x-z)^{\lambda}(\bar{x}-\bar{z})^{+\frac{1}{2} m+\frac{i}{2} \varrho+\frac{1}{2}(\lambda+\mu)} \times \\
& \times\left[-2 i \frac{\partial}{\partial \bar{x}}\right]^{\frac{1}{2}(\lambda+\mu)}(\bar{x}-\bar{z})^{-\frac{1}{2} m-\frac{i}{2} e-\frac{1}{2}(\lambda-\mu)} .
\end{aligned}
$$

Taking products of two such differential operators yields further covariant operators. In the case $\lambda=\mu=1$ we obtain:

$$
\begin{aligned}
& T(z)_{m \rightarrow m+2}=-2 i\left[\left(\frac{1}{2} m-\frac{i}{2} \varrho+1\right)(\bar{x}-\bar{z})+|x-z|^{2} \frac{\partial}{\partial x}\right], \\
& T(z)_{m+2 \rightarrow m}=-2 i\left[-\left(\frac{1}{2} m+\frac{i}{2} \varrho\right)(x-z)+|x-z|^{2} \frac{\partial}{\partial x}\right] .
\end{aligned}
$$

These expressions lead to

$$
\begin{aligned}
& \Gamma_{0}(m \rightarrow m+2)=-i\left[\left(\frac{1}{2} m-\frac{i}{2} \varrho+1\right) \bar{x}+\left(1+|x|^{2}\right) \frac{\partial}{\partial x}\right], \\
& \Gamma_{3}(m \rightarrow m+2)=-i\left[-\left(\frac{1}{2} m-\frac{i}{2} \varrho+1\right) \bar{x}+\left(1-|x|^{2}\right) \frac{\partial}{\partial x}\right], \\
& \Gamma_{0}(m+2 \rightarrow m)=-i\left[-\left(\frac{1}{2} m+\frac{i}{2} \varrho\right) x+\left(1+|x|^{2}\right) \frac{\partial}{\partial \bar{x}}\right], \\
& \Gamma_{3}(m+2 \rightarrow m)=-i\left[\left(\frac{1}{2} m+\frac{i}{2} \varrho\right) x+\left(1-|x|^{2}\right) \frac{\partial}{\partial \bar{x}}\right] .
\end{aligned}
$$


As a first example we study a representation with [1]

$$
\varrho=0, \quad m=+1 .
$$

This is a case where $U_{a}$ can be taken irreducible, the representation

$$
\varrho=0, \quad m=-1
$$

is equivalent to it. According to the two possible choices of $m$ we can build two different canonical basis:

$$
\begin{aligned}
f_{p}^{j}(x)^{(+)}= & {\left[\frac{(2 j+1)}{\pi} \cdot \frac{\left(j+\frac{1}{2}\right) !\left(j-\frac{1}{2}\right) !}{(j+p) !(j-p) !}\right]^{\frac{1}{2}} \cdot \varkappa_{1, \varrho}^{(j)} \times } \\
& \times\left(1+|x|^{2}\right)^{i \frac{\varrho}{2}-j-1} I_{+\frac{1}{2}, p}^{(j)}(x,-\bar{x})
\end{aligned}
$$

or

$$
\begin{aligned}
f_{p}^{j}(x)^{(-)}= & {\left[\frac{(2 j+1)}{\pi} \cdot \frac{\left(j+\frac{1}{2}\right) !\left(j-\frac{1}{2}\right) !}{(j+p) !(j-p) !}\right]^{\frac{1}{2}} \cdot \varkappa_{1, \varrho}^{(j)} \times } \\
& \times\left(1+|x|^{2}\right)^{i \frac{o}{2}-j-1} I_{-\frac{1}{2}, p}^{(j)}(x,-\bar{x}) .
\end{aligned}
$$

We define a unitary operator by

$$
V f_{p}^{j}(x)^{(-)}=-i \cdot f_{p}^{j}(x)^{(+)} .
$$

Then we define $\Gamma_{\mu}$ as the differential operators introduced above multiplied with $V$. This makes $\Gamma_{0}$ diagonal on the basis $f_{p}^{j}(x)^{(+)}$. We make use of the series expansions for the principal function $I$ and obtain $\left(f_{p}^{j}=f_{j}^{i}(x)^{(+)}\right)$

$$
\begin{aligned}
& \Gamma_{0} f_{p}^{j}=+\left(j+\frac{1}{2}\right) f_{p}^{j}, \\
& \Gamma_{3} f_{p}^{j}=-\frac{1}{2}\left\{\left[(j+1)^{2}-p^{2}\right]^{\frac{1}{2}} f_{p}^{j+1}+\left[j^{2}-p^{2}\right]^{\frac{1}{2}} f_{p}^{j-1}\right\} .
\end{aligned}
$$

We shall continue the discussion of this example and others in section $\mathrm{V}$. We emphasize that our differential operators satisfy the condition

$$
\begin{aligned}
\left|m_{1}-m_{2}\right| & =2, \\
\varrho_{1} & =\varrho_{2},
\end{aligned}
$$

which was required by the theorem of GELFAND and YAGLOM if applied to the principal series. The explicit form of the operators $\Gamma_{0}$ and $\Gamma_{3}$ as matrices in the canonical basis in the particular example considered above coincides with the analogous expressions of GELFAND and YAGLOM (see [5], page 421). 
We mention finally that $\Gamma_{3}$ can be obtained from $\Gamma_{0}$ by means of the commutator

$$
\Gamma_{3}=-i\left[\Gamma_{0}, F_{3}\right] \text {. }
$$

$F_{3}$ is one of the generators of $S L(2, C)$ and is given in both differential operator and matrix form in appendix, subsection 4.

\section{Proof of the Differential Operator Forms}

Though we need in principle only prove that the forms of the covariant operators $T(z)$ given above satisfy the correct covariance requirements, a derivation of these forms is of fundamental interest since it can show us where the origin of restrictions like

lies.

$$
\begin{aligned}
\left|m_{1}-m_{2}\right| & =2 \cdot \min (\lambda, \mu), \\
\varrho_{1} & =\varrho_{2}
\end{aligned}
$$

We mention that in a case when $T(z)$ transforms as a unitary representation $S(m, \varrho)$ of the principal series, we can define $T(z)$ as an integral operator in $\mathscr{L}^{2}\left(Z_{2}\right)$,

$$
F(z)=(h, T(z) f)=\int K\left(z, z_{1}, z_{2}\right) \bar{h}\left(z_{1}\right) f\left(z_{2}\right) d z_{1} d z_{2}
$$

where $K$ is unique up to a constant [10]. If $f$ and $h$ are of bicompact support in $z$ and possess derivatives of arbitrary order, the integral can be shown to exist in the sense of absolute convergence.

We can try to generalize this construction of $T(z)$ in order to include also spinorial transformation behaviour of $T(z)$. The kernel $K$ can formally be determined by solving some functional equations (see the discussion in Ref. [11]). It is again unique up to a factor. The question whether the integral converges is, however, not trivial.

In fact we can show that the convergence behaviour lies outside, yet on the boundary of the domain of conditional convergence. Infinitesimal changes in the parameters $\varrho$ allow us to approach the integral from inside the domain of convergence. The integral tends to infinity. But dividing through an appropriate function of the Casimir invariant leaves us a correctly transforming, finite, "renormalized" integral. It possesses the form

$$
\widetilde{F}(z)=\int \bar{h}\left(z_{1}\right) P\left(z, z_{1}, \frac{\partial}{\partial z_{1}}, \bar{z}_{1}, \frac{\partial}{\partial \bar{z}_{1}}\right) f\left(z_{1}\right) d z_{1}
$$

where $P$ is a polynomial of $z, \bar{z}, z_{1}, \bar{z}_{1}, \frac{\partial}{\partial z_{1}}, \frac{\partial}{\partial \bar{z}_{1}}$.

The kernel $K$ is

$$
\begin{aligned}
K\left(z, z_{2}, z_{1}\right)= & \left(z_{1}-z_{2}\right)^{-A}\left(z_{1}-z\right)^{-A_{1}}\left(z_{2}-z\right)^{-A_{2}} \times \\
& \times\left(\bar{z}_{1}-\bar{z}_{2}\right)^{-B}\left(\bar{z}_{1}-\bar{z}\right)^{-B_{1}}\left(\bar{z}_{2}-\bar{z}\right)^{-B_{2}}
\end{aligned}
$$


with

$$
\begin{aligned}
& A=\frac{1}{2}\left(\lambda+\lambda_{1}-\lambda_{2}\right)+1, \quad B=\frac{1}{2}\left(\mu+\mu_{1}-\mu_{2}\right)+1, \\
& A_{1}=\frac{1}{2}\left(-\lambda+\lambda_{1}+\lambda_{2}\right)+1, \quad B_{1}=\frac{1}{2}\left(-\mu+\mu_{1}+\mu_{2}\right)+1, \\
& A_{2}=-\frac{1}{2}\left(\lambda+\lambda_{1}+\lambda_{2}\right)-1, \quad B_{2}=-\frac{1}{2}\left(\mu+\mu_{1}+\mu_{2}\right)-1,
\end{aligned}
$$

and

$$
\lambda_{i}=-\frac{1}{2} m_{i}+\frac{i}{2} \varrho_{i}-1, \quad \mu_{i}=+\frac{1}{2} m_{i}+\frac{i}{2} \varrho_{i}-1 .
$$

Since $\lambda, \mu$ satisfy $\lambda+\mu>0$, the functions

$$
\begin{aligned}
& \Phi_{z}\left(z_{1}\right)=z_{1}{ }^{-A_{1}} \bar{z}_{1}-B_{1} f\left(z_{1}+z\right), \\
& \Psi_{z}\left(z_{2}\right)=\bar{z}_{2}-\bar{A}_{2} z_{2}-\bar{B}_{2} h\left(z_{2}+z\right)
\end{aligned}
$$

are bounded and integrable. They possess Fourier transforms

$$
\begin{aligned}
& \widetilde{\Phi}_{z}(w)=(2 \pi)^{-1} \int \Phi_{z}\left(z_{1}\right) e^{i \operatorname{Re}\left(z_{1} \bar{w}\right)} d z_{1}, \\
& \widetilde{\Psi}_{z}(w)=(2 \pi)^{-1} \int \Psi_{z}\left(z_{2}\right) e^{i \operatorname{Re}\left(z_{2} \bar{u}\right)} d z_{2} .
\end{aligned}
$$

We assume now that

$$
B-A \geqq 0,
$$

then the integral

$$
\int e^{-i \operatorname{Re}(z \bar{w})} z^{-A} \bar{z}^{-B} d z
$$

exists, provided $\operatorname{Re} A<+1$, and yields

$$
\pi \cdot 2^{2-A-B} e^{-i \frac{\pi}{2}(B-A)} \cdot \Gamma(-A+1) \cdot[\Gamma(B)]^{-1} w^{B-1} \bar{w}^{A-1} .
$$

This allows us to express $F(z)$ by

$$
\begin{aligned}
F(z)= & \int \overline{\Psi_{z}\left(z_{2}\right)} \Phi_{z}\left(z_{1}\right)\left(z_{1}-z_{2}\right)^{-A}\left(\bar{z}_{1}-\bar{z}_{2}\right)^{-B} d z_{1} d z_{2} \\
= & \pi \cdot 2^{2-A-B} e^{-i \frac{\pi}{2}(B-A)} \cdot \Gamma(-A+1) \cdot[\Gamma(B)]^{-1} \times \\
& \times \int \overline{\widetilde{\Psi}_{z}(w)} w^{B-1} \bar{w}^{d-1} \widetilde{\Phi}_{z}(w) d w .
\end{aligned}
$$

If we want $T(z)$ to become a differential operator, we must satisfy the conditions

$$
\begin{aligned}
& B-1 \geqq 0, \text { integer }, \\
& A-1 \geqq 0, \text { integer } .
\end{aligned}
$$

Since $\operatorname{Re} A<+1$ was postulated above we have two contradictory requirements. With small changes in the $\varrho$ 's we can, however, reach the point $A=1$. The divergent expression $\Gamma(-A+1)$ is factored out and can be eliminated by the renormalization. This means we define a new function

$$
\widetilde{F}(z)=\int \overline{\widetilde{\Psi}_{z}(w)} w^{B-1} \widetilde{\Phi}_{z}(w) d w
$$


and put $A=+1$ everywhere. This implies

Since

$$
\begin{aligned}
\lambda=\frac{1}{2}\left(m_{1}-m_{2}\right), \quad \mu & =-\frac{1}{2}\left(m_{1}-m_{2}\right)+2 n, \\
n=B-1 & =\frac{1}{2}(\lambda+\mu), \\
\varrho_{1} & =\varrho_{2} .
\end{aligned}
$$

$$
\begin{aligned}
& \lim _{|w| \rightarrow \infty}|w|^{n}\left|\widetilde{\Phi}_{z}(w)\right|=0, \\
& \lim _{|w| \rightarrow \infty}|w|^{n}\left|\widetilde{\Psi}_{z}(w)\right|=0
\end{aligned}
$$

the integral $F(z)$ is finite. Using the Plancherel formula we obtain

$$
\begin{aligned}
\tilde{F}(z)=\int \bar{h}(x)(x-z)^{-A_{2}} & (\bar{x}-\bar{z})^{-B_{2}} \times \\
& \times\left(-2 i \frac{\partial}{\partial \bar{x}}\right)^{B-1}(x-z)^{-A_{1}}(\bar{x}-\bar{z})^{-B_{1}} f(x) d x .
\end{aligned}
$$

If $B_{1}$ is not a negative integer or zero,

$$
-B_{2}-B_{1}-B+1=\frac{1}{2}(\mu-\lambda)
$$

must be non-negative, in order that $T(z)$ becomes a polynomial in $(x-z)$. Therefore we have a final condition:

$$
\mu \geqq \lambda \text {. }
$$

If $A-B \geqq 0$, we need only replace

$$
A \text { 's by } B \text { 's, } x \text { by } \bar{x}, \approx \text { by } \bar{z}
$$

and vice versa. In that case we get a final condition

$$
\lambda \geqq \mu
$$

Inserting the values for $A$ 's and $B$ 's leads to the operators given in subsection 2 .

\section{The Canonical Pseudo-Basis}

1. Points of the Space $S L(2, C) / K$ Described by Elemenis of $S U(1,1)$

As in section II we consider the subgroup $K$ of triangular matrices $k$,

and consider the restclasses

$$
k=\left(\begin{array}{ll}
\lambda^{-1} & \mu \\
0 & \lambda
\end{array}\right)
$$

$$
S L(2, C) / K \text {. }
$$

In some of the restclasses we can find an element $v \in S U(1,1)$, in almost all of the remaining restclasses there are elements of the type $\varepsilon \cdot v$, $v \in S U(1,1)$,

$$
\varepsilon=i \sigma_{1}=\left(\begin{array}{ll}
0 & i \\
i & 0
\end{array}\right)
$$


In fact, almost all elements $a \in S L(2, C)$ can be decomposed as

$$
a=k \cdot v
$$

or as

$$
a=k \cdot \varepsilon \cdot v .
$$

Thus we can characterize points in the homogeneous space by points of two groups $S U(1,1)_{+}$and $S U(1,1)_{-}$. As earlier $v$ and $\varepsilon \cdot v$ are not uniquely determined. In fact any $\gamma v$ (or $\varepsilon \gamma v$ ) is in the same restclass with $v$ (or $\varepsilon \cdot v$ ), when $\gamma$ is defined as

Obviously is $\gamma \varepsilon=\varepsilon \gamma^{-1}$.

$$
\gamma=\left(\begin{array}{ll}
e^{i \omega} & 0 \\
0 & e^{-i \omega}
\end{array}\right) \text {. }
$$

We construct a Hilbert space $\mathfrak{G}_{m}$ of pairs of square integrable functions

such that

$$
\varphi=\left\{\varphi_{+}, \varphi_{-}\right\}
$$

$$
\|\varphi\|^{2}=\int\left[\left|\varphi_{+}(v)\right|^{2}+\left|\varphi_{-}(v)\right|^{2}\right] d \mu(v) .
$$

$\varphi_{ \pm}(v)$ has to satisfy the subsidiary conditions:

$$
\begin{aligned}
& \varphi_{+}(\gamma v)=e^{+i m \omega} \varphi_{+}(v), \\
& \varphi_{-}(\gamma v)=e^{-i m \omega} \varphi_{-}(v) .
\end{aligned}
$$

The Hilbert space $\mathfrak{G}_{m}$ is the direct orthogonal sum

$$
\mathfrak{S}_{m}=\mathscr{L}_{m}^{2}(V) \oplus \mathscr{L}_{-m}^{2}(V),
$$

where $\mathscr{L}_{m}^{2}(V)$ is made up of functions $\varphi_{+}(v)$ and $\mathscr{L}_{-m}^{2}(V)$ of functions $\varphi_{-}(v)$.

Let us find now the connection between the parameters $z$ and $v, \varepsilon \cdot v$.

In the case $z=z(v)$ we have

with the solution

$$
\left(\begin{array}{ll}
1 & 0 \\
z & 1
\end{array}\right)=\left(\begin{array}{cc}
\lambda-1 & \mu \\
0 & \lambda
\end{array}\right)\left(\begin{array}{ll}
v_{11} & v_{12} \\
v_{21} & v_{22}
\end{array}\right)
$$

This implies

$$
\begin{aligned}
z & =v_{21} \cdot v_{22}^{-1}, \\
\lambda & =|\lambda| \cdot e^{i \omega} \\
& =e^{i \omega}\left(1-|z|^{2}\right)^{\frac{1}{2}}=v_{22}^{-1} .
\end{aligned}
$$

$$
|z(v)|<1 .
$$

In the case $z=z(\varepsilon v)$ we find from

$$
\begin{gathered}
\left(\begin{array}{ll}
1 & 0 \\
z & 1
\end{array}\right)=\left(\begin{array}{cc}
\lambda^{-1} & \mu \\
0 & \lambda
\end{array}\right)\left(\begin{array}{ll}
0 & i \\
i & 0
\end{array}\right)\left(\begin{array}{ll}
v_{11} & v_{12} \\
v_{21} & v_{22}
\end{array}\right), \\
z=v_{11} \cdot v_{12}^{-1}, \\
\lambda=e^{i \omega}\left(|z|^{2}-1\right)^{\frac{1}{2}}=\left(i v_{12}\right)^{-1} .
\end{gathered}
$$


In this case $z$ satisfies

$$
|z(\varepsilon v)|>1 \text {. }
$$

In both cases $\omega$ is an arbitrary phase.

Writing

$$
f(z)=f(z) \cdot \theta(1-|z|)+f(z) \cdot \theta(|z|-1)
$$

we obtain a decomposition of $\mathscr{L}^{2}(Z)$

$$
\mathscr{L}^{2}(Z)=\mathscr{L}_{+}^{2}(Z) \oplus \mathscr{L}^{2}(Z) .
$$

We map $\mathscr{L}^{2}{ }_{+}(Z)$ on $\mathscr{L}^{2}{ }_{m}(V)$ and $\mathscr{L}^{2}{ }_{-}(Z)$ on $\mathscr{L}^{2}{ }_{-m}(V)$ by the relation

$$
\begin{aligned}
& \varphi_{+}(v)=\pi^{\frac{1}{2}} \alpha(v) \cdot f(z) \cdot \theta(1-|z|), \\
& \varphi_{-}(v)=\pi^{\frac{1}{2}} \alpha(\varepsilon v) \cdot f(z) \cdot \theta(|z|-1) .
\end{aligned}
$$

We can easily show that this mapping is in accord with the subsidiary conditions imposed on $\varphi_{ \pm}(v)$.

We introduce the parameters for $v$ :

$$
\begin{array}{cc}
v_{11}=s^{\frac{1}{2}} e^{i \theta_{1}}, & v_{12}=(s-1)^{\frac{1}{2}} e^{i \theta_{2}}, \\
1 \leqq s<\infty, & 0 \leqq \theta_{1}, \theta_{2}<2 \pi .
\end{array}
$$

We normalize the invariant measure by

$$
d \mu(v)=\frac{1}{4 \pi^{2}} d s d \theta_{1} d \theta_{2}
$$

The parameters $s, \theta_{1}, \theta_{2}$ are related with $z, \omega$ by the following formulae.

If $|z|<1$ :

$$
\begin{gathered}
s=\left(1-|z|^{2}\right)^{-1}, \theta_{1}=\omega, \theta_{2}=\omega-\psi, \psi=\arg z, \\
d \mu(v)=\frac{1}{2 \pi^{2}}|\lambda|^{-4} \cdot d z d \omega,
\end{gathered}
$$

and if $|z|>1$ :

$s=|z|^{2} \cdot\left(|z|^{2}-1\right)^{-1}, \quad \theta_{1}=-\omega+\psi-\frac{\pi}{2}, \quad \theta_{2}=-\omega-\frac{\pi}{2}, \quad \psi=\arg z$,

Since in both cases

$$
d \mu(v)=\frac{1}{2 \pi^{2}} \cdot|\lambda|^{-4} \cdot d z d \omega
$$

we obtain

$$
\alpha(v)=(\alpha(\varepsilon v) \Rightarrow|\lambda|-m-i \varrho+2 \cdot \lambda+m
$$

With the notation

$$
\begin{aligned}
& \int\left|\varphi_{+}(v)\right|^{2} d \mu(v)=\int_{|z|<1}|f(z)|^{2} d z, \\
& \int\left|\varphi_{-}(v)\right|^{2} d \mu(v)=\int_{|z|>1}|f(z)|^{2} d z .
\end{aligned}
$$

$$
\zeta= \pm 1, \quad \tau=\frac{1-\zeta}{2}
$$


we may finally write

where

$$
U_{\alpha} \varphi_{\varrho}(v)=\frac{\alpha\left(\varepsilon^{\tau} v a\right)}{\alpha\left(\varepsilon^{\tau^{\prime}} v^{\prime}\right)} \varphi_{\varrho^{\prime}}\left(v^{\prime}\right)
$$

$$
\varepsilon^{\tau} v a=k \cdot \varepsilon^{\tau^{\prime}} v^{\prime}
$$

\section{The Canonical Pseudo-Basis in $\mathfrak{G}_{m}$}

A Hilbert space $\mathscr{L}^{2}(V)$ of square integrable functions on $S U(1,1)$ possesses a pseudo-basis which consists of the matrix elements $C_{p q}^{\chi}(v)$ of unitary representations $\chi$ of $S U(1,1)$ where $\chi$ is in the set:

$$
\chi= \begin{cases}\chi(\xi, \eta), & 0<\xi<\infty, \quad \eta=0, \frac{1}{2} \\ \chi(k, \pm), & k=1, \frac{3}{2}, 2, \ldots\end{cases}
$$

(see the explicit construction of $C_{p q}^{x}(v)$ for these cases in appendix, subsection 3 ). In both cases we shall use the notation of

$$
\begin{aligned}
& J=-\frac{1}{2}+i \xi \quad \text { in } \quad \chi(\xi, \eta), \\
& J=-k \text { in } \chi(k, \pm) .
\end{aligned}
$$

With the Fourier coefficients

$$
K(\varphi)_{p q}^{\chi}=\int \varphi(v) \overline{C_{p q}^{\chi}(v)} d \mu(v)
$$

the Plancherel theorem for $S U(1,1)$ takes the form $[7,8]$ :

$$
\begin{aligned}
\varphi(v)= & \int_{0}^{\infty} C(\xi, 0) d \xi \sum_{p, q=-\infty}^{+\infty} K(\varphi)_{p, q}^{(\xi, 0)} C_{p, q}^{(\xi, 0)}(v)+ \\
& +\int_{0}^{\infty} C\left(\xi, \frac{1}{2}\right) d \xi \sum_{p, q=-\infty}^{+\infty} K(\varphi)_{p, q}^{\left(\xi, \frac{1}{2}\right)} C_{p, q}^{\left(\xi, \frac{1}{2}\right)}(v)+ \\
& +\sum_{k=1, \frac{3}{2}, 2, \ldots}^{\infty} C(k)\left\{\sum_{q, p=k}^{+\infty} K(\varphi)_{p, q}^{(k,+)} C_{p, q}^{(k,+)}(v)+\right. \\
& \left.+\sum_{q, p=-k}^{\infty} K(\varphi)_{p, q}^{(k,-)} C_{p, q}^{(k,-)}(v)\right\}
\end{aligned}
$$

The right hand side converges in the mean square sense. The weights are

$$
\begin{aligned}
C(k) & =2 k-1, \\
C\left(\xi, \frac{1}{2}\right) & =2 \xi \operatorname{cth} \pi \xi, \\
C(\xi, 0) & =2 \xi \operatorname{th} \pi \xi .
\end{aligned}
$$


Both spaces $\mathscr{L}^{2}{ }_{m}(V)$ and $\mathscr{L}^{2}-m(V)$ are subspaces in $\mathscr{L}^{2}(V)$. The subsidiary conditions yield

which implies

$$
\begin{aligned}
K\left(\varphi_{\zeta}\right)_{p q}^{\chi} & =\int \varphi_{\zeta}(v) \overline{C_{p q}^{\chi}(v)} d \mu(v) \\
& =\int \varphi_{\zeta}(\gamma v) \overline{C_{p q}^{\chi}(\gamma v)} d \mu(v) \\
& =e^{i \omega(\zeta m-2 p)} \cdot K\left(\varphi_{\zeta}\right)_{p q}^{\chi}
\end{aligned}
$$

$$
K\left(\varphi_{\zeta}\right)_{p q}^{\chi}=0, \text { or } \quad p=\zeta \frac{m}{2} \text {. }
$$

This reduces the basis of $\mathscr{L}^{2}(V)$ to the basis of $\mathscr{L}^{2}{ }_{m}(V)$ and we get

$$
\begin{aligned}
\varphi_{\zeta}(v)= & \int_{0}^{\infty} C(\xi, 0) d \xi \sum_{q=-\infty}^{+\infty} K\left(\varphi_{\zeta}\right)_{\zeta \frac{m}{2}, q}^{(\xi, 0)} C_{\zeta \frac{m}{2}, q}^{(\xi, 0)}(v)+ \\
& +\sum_{k=1,2, \ldots}^{j_{0}} C(k) \sum_{|q|=k, k+1, \ldots}^{+-\infty} K\left(\varphi_{\zeta}\right)_{\zeta \frac{m}{2}, q}^{(k, \zeta \sigma)} C_{\zeta \frac{m}{2}, q}^{(k, \zeta \sigma)}(v),
\end{aligned}
$$

if $m$ is even and a similar expression if $m$ is odd. We recall that

$$
\sigma=\operatorname{sign} m, \quad j_{0}=\left|\frac{m}{2}\right|, \quad \operatorname{sign} q=\zeta \cdot \sigma .
$$

The sum over $k$ is finite, this is due to the fact that in $C_{q, p}^{(k, \pm)}$ both $|q|$ and $|p|$ are not smaller than $k$.

In $\mathscr{L}_{m}^{2}(V)$ we define the pseudo-basis by

$$
\left.\begin{array}{l}
C_{\frac{m}{2}, q}^{(\xi, 0)}(v), \quad 0<\xi<\infty, \\
(2 k-1)^{\frac{1}{2}} \cdot C_{\frac{m}{2}, q}^{(k, \sigma)}(v), \quad k=1,2,3, \ldots
\end{array}\right\} \text { for even } m,
$$

Together with a corresponding pseudo-basis in $\mathscr{L}_{-m}^{2}(V)$ these vectors define the canonical basis in $\mathfrak{G}_{m}$. The basis vectors of the discrete series are normalized to one.

It is obvious that each $\mathscr{L}_{\zeta m}^{2}(V)$ is invariant with respect to $S U(1,1)$

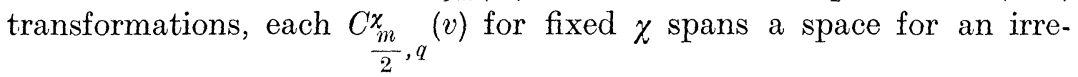
ducible unitary representation of $S U(1,1)$. The decomposition of $\mathfrak{G}_{m}$ into $\mathscr{L}_{m}^{2}(V) \oplus \mathscr{L}^{2}{ }_{-m}(V)$ removes the degeneracy in the continuous series.

23 Commun. math. Phys., Vol. 6 


\section{The Canonical Pseudo-Basis in $\mathscr{L}^{2}(Z)$}

We make use of the relation between $f(z)$ and $\varphi_{\zeta}(v)$ to find the pseudobasis in $\mathscr{L}^{2}(Z)$. We obtain basis vectors $f_{p}^{\chi, \zeta}(Z)$ which can be expressed by the principal function $I_{p q}^{J}(z, \bar{z})$ (see appendix, subsection 1) as follows:

$$
\begin{aligned}
& f_{p}^{(\xi, \eta),+}(z)=+\pi^{-\frac{1}{2}}\left(1-|z|^{2}\right)^{i \frac{\varrho}{2}-J-1} \cdot I_{\frac{m}{2}, p}^{J}(z, \bar{z}) \cdot \theta(1-|z|), \\
& f_{p}^{(\xi, \eta),-}(z)=+i^{m} \cdot \pi^{-\frac{1}{2}}\left(|z|^{2}-1\right)^{i \frac{\varrho}{2}-J-1} I_{\frac{m}{2}, p}^{J}(z, \bar{z}) \cdot \theta(|z|-1),
\end{aligned}
$$

where

$$
\begin{aligned}
& J=-\frac{1}{2}+i \xi, \\
& f_{p}^{(k, \sigma)}(z)=+\left[\frac{(2 k-1)}{\pi} \cdot \frac{(k+|p|-1) !\left(j_{0}-k\right) !}{\left(k+j_{0}-1\right) !\left(\left|p_{0}\right|-k\right) !}\right]^{\frac{1}{2}} \times \\
& \times\left(1-|z|^{2}\right)^{i \frac{\varrho}{2}-J-1} \cdot I_{\frac{m}{2}, p}^{J}(z, \bar{z}) \cdot \theta(1-|z|), \\
& f_{p}^{(k,-\sigma)}(z)=+i^{m}\left[\frac{(2 k-1)}{\pi} \cdot \frac{(k+|p|-1) !\left(j_{0}-k\right) !}{\left(k+j_{0}-1\right) !\left(\left|p_{0}\right|-k\right) !}\right]^{\frac{1}{2}} \times \\
& \times\left(|z|^{2}-1\right)^{i \frac{\varrho}{2}-J-1} \cdot I_{\frac{m}{2}, p}^{J}(z, \bar{z}) \cdot \theta(|z|-1),
\end{aligned}
$$

where in the last two cases

$$
J=-k, \quad \operatorname{sign} p= \pm \sigma .
$$

\section{The Change of Basis in $\mathscr{L}^{2}(Z)$}

The transition from the canonical basis to the canonical pseudobasis and vice versa can be performed by means of coefficients

which are defined as

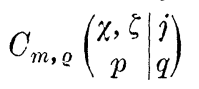

$$
C_{m, \varrho}\left(\begin{array}{c}
\chi, \zeta \mid \\
p
\end{array} \mid \begin{array}{l}
j \\
q
\end{array}\right)=\int \overline{f_{p}^{x, \zeta}(z)} f_{q}^{j}(z) d z=\delta_{p q} \cdot \int \overline{f_{q}^{x, \zeta}(z)} f_{q}^{j}(z) d z .
$$

They satisfy certain orthogonality relations which can be read off the orthogonality relations for the elements of the basis and pseudo-basis. We give an example:

Here $\delta_{\chi x^{\prime}}$ means:

$$
\sum_{j=j_{0}}^{\infty} \sum_{q=-j}^{+j} C_{m, \varrho}\left(\begin{array}{c}
\chi, \zeta \mid j \\
p
\end{array}\right) \overline{C_{m, \varrho}\left(\begin{array}{c}
\chi^{\prime}, \zeta^{\prime} \mid j \\
p^{\prime} \mid q
\end{array}\right)}=\delta_{\chi \chi^{\prime}} \delta_{p p^{\prime}} \delta_{\zeta \zeta^{\prime}} .
$$

$$
\delta_{\chi \chi^{\prime}}=\left\{\begin{array}{l}
0, \text { if } \chi \text { and } \chi^{\prime} \text { belong to different series, } \\
C^{-1}(\xi, \eta) \delta\left(\xi-\xi^{\prime}\right) \delta_{\eta \eta^{\prime}} \text { if } \chi=\chi(\xi, \eta), \quad \chi^{\prime}=\chi\left(\xi^{\prime}, \eta^{\prime}\right), \\
\delta_{k k^{\prime}} \delta_{\sigma \sigma^{\prime}}, \text { if } \chi=\chi(k, \sigma), \quad \chi^{\prime}=\chi\left(k^{\prime}, \sigma^{\prime}\right) .
\end{array}\right.
$$

Since these coefficients are of no further use we avoid the evaluation of the complicated integrals. 


\section{Mass Spectra}

\section{The Reduced $\Gamma$ Matrices}

We denote the Hilbert space which contains $\Psi$ and carries the representation $U_{a}$ by $\mathfrak{G}$. We assume throughout that $\mathfrak{S}$ is a finite direct sum, e.g. of the type

$$
\mathfrak{G}=\sum_{S=1}^{n} \oplus \mathscr{L}^{2}\left(Z_{S}\right)
$$

Each $S$ labels an irreducible unitary representation of $S L(2, C)$ in the space $\mathscr{L}^{2}\left(Z_{S}\right)$. Let the projection operator on the $S U(2)$ invariant subspace of spin $j$ in $\mathscr{L}^{2}\left(Z_{S}\right)$ be denoted $P_{j}(S)$. We define two other projection operators

$$
\begin{gathered}
P_{j}=\sum_{S=1}^{n} \oplus P_{j}(S), \\
P(S)=\sum_{j=j_{0}(S)}^{\infty} \oplus P_{j}(S) .
\end{gathered}
$$

We introduce the transition operators which map the spin space $j$ of $\mathscr{L}^{2}\left(Z_{S_{1}}\right)$ on the spin space $j$ of $\mathscr{L}^{2}\left(Z_{S_{2}}\right)$ by

$$
\begin{aligned}
E_{S_{1} S_{2}}^{j} E_{S_{3} S_{4}}^{j^{\prime}} & =\delta_{j j^{\prime}} \delta_{S_{2} S_{\mathbf{3}}} E_{S_{1} S_{\mathbf{4}}}^{j_{4}}, \\
E_{S S}^{j} & =P_{j}(S) .
\end{aligned}
$$

Since $P_{j} \Gamma_{0}=\Gamma_{0} P_{j}, \Gamma_{0}$ reduces to blocks $\left(\Gamma_{0}\right)_{j}$

$$
\begin{aligned}
\left(\Gamma_{0}\right)_{j} & =P_{j} \Gamma_{0} P_{j}, \\
\Gamma_{0} & =\sum_{j} \oplus\left(\Gamma_{0}\right)_{j} .
\end{aligned}
$$

Each block $\left(\Gamma_{0}\right)_{j}$ can be decomposed further by

$$
\left(\Gamma_{0}\right)_{j}=\sum_{S_{1}, S_{2}} \oplus \beta_{S_{1} S_{2}}^{j_{2}} E_{S_{1} S_{2}}^{j_{2}}
$$

By this formal procedure we get a matrix $\beta_{S_{1} S_{2}}^{j}$, the reduced $\left(\Gamma_{0}\right)_{j}$ matrix (in the WIGNER-EcKarT sense), which is independent of $p$. This reduced matrix is finite dimensional and hermitean, it has eigenvalues $M(j)$ belonging to eigenvectors $\alpha_{S}^{j}(M)$.

Now we build vectors $\Psi_{p}^{j}(M)$ in $\mathfrak{G}$, such that

$$
P(S) \Psi_{p}^{j}(M)=\alpha_{S}^{j}(M) f_{p}^{j}\left(z_{S}\right)
$$

If $p$ varies from $-j$ to $+j, M(j)$ over all eigenvalues of $\beta^{j}$ and $j$ over all possible values, we get a set of vectors $\left\{\Psi_{p}^{j}(M)\right\}$ which are complete and orthogonal in $\mathfrak{S}$. Applying $U_{a(p)}$ to them we get a complete set of timelike solutions.

Apart from the complications due to the continuous series we can apply the same procedure to $\Gamma_{3}$ and the $S U(1,1)$ invariant spaces 23* Commun. math. Phys., Vol. 6 
$\mathfrak{S}^{\chi, \zeta}$ into which $\mathfrak{S}$ can be decomposed. We get again a finite hermitean matrix $\beta_{S_{1} S_{3}}^{\chi, \zeta}$ and eigenvalues $\lambda(\chi, \zeta)$.

The computation of the $\beta$ matrices and their eigenvalues is the last problem to be tackled.

\section{Two Simple Cases}

It turns out that the case of irreducible representations $U_{a}$ is particularly simple. In that case $\beta$ are numbers. In section III we started the discussion of one such example, viz.

Another case,

$$
m= \pm 1, \quad \varrho=0
$$

$$
m=0, \quad \varrho= \pm i
$$

belongs to the supplementary series. Both yield identical matrix elements of $\Gamma_{0}$ and $\Gamma_{3}{ }^{3}$ in the canonical basis (see section III and Ref. [5] page 421):

$$
\begin{gathered}
\Gamma_{0} f_{p}^{j}=+\left(j+\frac{1}{2}\right) f_{p}^{j}, \\
\Gamma_{3} f_{p}^{j}=-\frac{1}{2}\left\{\left[(j+1)^{2}-p^{2}\right]^{\frac{1}{2}} f_{p}^{j+1}+\left[j^{2}-p^{2}\right]^{\frac{1}{2}} f_{p}^{j-1}\right\} .
\end{gathered}
$$

It is remarkable that $\Gamma_{3}$ can be diagonalized immediately without explicit use of the pseudo-basis. This is of particular interest, since by the same method we find also the solutions on the lightcone. We proceed as follows.

The matrix $\Gamma_{3}$ depends only on $|p|$. We denote

and for fixed $p$

$$
n=j-|p| \text {, }
$$

This yields

$$
f=\sum_{n=0}^{\infty} \psi_{n} f_{p}^{j}
$$

$$
[(n+1)(n+2|p|+1)]^{\frac{1}{2}} \psi_{n+1}+2 \mu \psi_{n}+[n(n+2|p|)]^{\frac{1}{2}} \psi_{n-1}=0,
$$

with the eigenvalue $\mu$ of $\Gamma_{3}$.

The Hilbert space $l^{2}$ of sequences $\left\{\psi_{n}\right\}$,

$$
\|\psi\|^{2}=\sum_{n=0}^{\infty}\left|\psi_{n}\right|^{2}<\infty
$$

is isomorphic to the Hilbert space $\mathscr{F}$ of entire functions with the norm

$$
\|g\|^{2}=\frac{2}{\pi} \int K_{2|p|}(2|z|) \cdot|z|^{2|p|}|g(z)|^{2} d z .
$$

$K$ is a cylinder function with imaginary argument (Ref. [12]) equ. 8. 407).

3 The matrix elements are identical concerning the dependence on $j$ and $p$, the permitted values of $j$ and $p$ differ, however, in both cases. 
The isomorphism between $l^{2}$ and $\mathscr{F}$ can be established by

$$
g(z)=\sum_{n=0}^{\infty} \frac{\psi_{n}}{[n !(n+2|p|) !]^{\frac{1}{2}}} z^{n} .
$$

This yields (see Ref. [12] equ. 6. 561. 16)

$$
\|g\|^{2}=\sum_{n=0}^{\infty}\left|\psi_{n}\right|^{2}=\|\|^{2}
$$

In the space $\mathscr{F}$ the eigenvalue equation for $\Gamma_{3}$ takes the form of the confluent hypergeometric equation

$$
z \cdot g^{\prime \prime}(z)+(2|p|+1) g^{\prime}(z)+(z+2 \mu) g(z)=0 \text {. }
$$

There is one entire solution for any $\mu,-\infty<\mu<+\infty$,

$$
\begin{aligned}
g(z)= & e^{i z} \Phi\left(|p|+\frac{1}{2}-i \mu, 2|p|+1 ;-2 i z\right) \\
= & 4^{-|p|}\left[B\left(|p|+i \mu+\frac{1}{2},|p|-i \mu+\frac{1}{2}\right)\right]^{-1} \times \\
& \times \int_{-1}^{+1}(1+t)^{|p|-i \mu-\frac{1}{2}}(1-t)^{|p|+i \mu-\frac{1}{2}} e^{-i z t} d t .
\end{aligned}
$$

$\Phi$ is Kummer's series (Ref. [12], equ. 9. 210. 1), $B$ is Euler's beta function (Ref. [12], equs. 8. 38) and the equality of the two expressions is asserted in Ref. [12] equ. 9. 211. 1.

The integral representation of $g(z)$ is particularly convenient to expand $g(z)$ in powers of $z$ and to obtain $\psi_{n}(\mu)$. We can show that this yields $\psi_{n}(\mu)$ with the asymptotic property

$$
\lim _{n \rightarrow \infty} n^{\frac{1}{2}}\left|\psi_{n}(\mu)\right|=\text { constant } \neq 0
$$

so that

$$
\|g\|^{2}=\sum_{n=0}^{\infty}\left|\psi_{n}(\mu)\right|^{2}=\infty
$$

The spectrum of $\Gamma_{3}$ contains no proper eigenvalue!

The Casimir operator $Q$ of $S U(1,1)$, $Q=F_{+} F_{-}+H_{3}-H_{3}^{2},\left(F_{ \pm}, H_{3}\right.$ as defined in appendix, subsection 4$)$. yields

$$
Q \psi_{n}(\mu)=\left(\mu^{2}+\frac{1}{4}\right) \psi_{n}(\mu)=-J(J+1) \psi_{n}(\mu)
$$

or

$$
J=-\frac{1}{2}+i \xi, \quad \xi=|\mu| .
$$

Each $J$ is twofold degenerate. If we understand $J$ to run over

$$
\begin{aligned}
& J=j_{0}+\text { non-negative integer } \\
& J=-\frac{1}{2}+\xi, \quad 0<\xi<\infty
\end{aligned}
$$

simultaneously, we may write the mass formula

valid for all $J$.

$$
M^{2}=\frac{x^{2}}{\left(J+\frac{1}{2}\right)^{2}}
$$

23a Commun. math. Phys., Vol. 6 
The sign of the energy in the timelike solutions is fixed and equal to $\operatorname{sign} \varkappa$. The spectrum is drawn in Fig. 1.

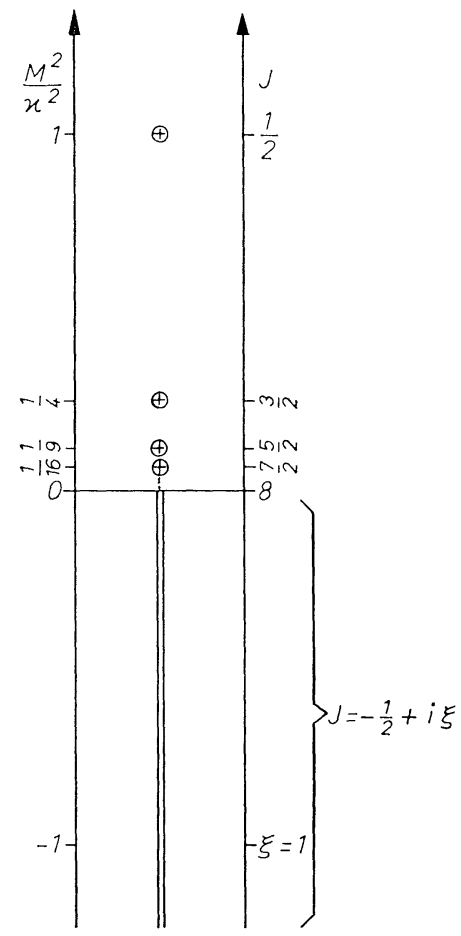

Fig. 1. $S=S( \pm 1,0)$

Let us consider now the solutions on the lightcone $M^{2}=0$, in which case the normal forms of the field equations can be defined as

$$
\left(\Gamma_{0}+\Gamma_{3} \mp \frac{1}{2}\right) \psi=0 \text {. }
$$

The two signs correspond to the future and the past half-cone,

$$
\operatorname{sign} \text { energy }= \pm \operatorname{sign} x \text {. }
$$

Just as above we can translate this equation into a differential equation operating on $\mathscr{F}$ and solve it. We obtain the entire solution

$$
\begin{aligned}
g \pm(z) & =e^{z}( \pm z)^{-|p|} J_{2|p|}(2 \sqrt{ \pm z}) \\
& =\frac{1}{2 \pi i} \int_{-\infty}^{(0,+)} t^{-2|p|-1} \exp \left(t+z\left(1 \mp t^{-1}\right)\right) d t .
\end{aligned}
$$

$J$ is a Bessel function, for the integral representation see Ref. [12], equ. 8.412.2. 
If $p$ runs over all possible values, the elements $g_{+}(z)$ (respectively $\left.g_{-}(z)\right)$ span an infinite dimensional space which carries an irreducible representation of the little group $U(1) \times T_{2}$, a so-called "continuous spin" representation. The point $M^{2}=0$ of the mass spectrum is twofold degenerate, one solution corresponds to the future cone, the other to the past cone. Concerning the degeneracy and the dimension of the representation of the little group, the solutions $M^{2}=0$ resemble the spacelike rather than the timelike solutions. This is true in general.

\section{The Class $S(m+1, \varrho) \oplus S(m-1, \varrho), m \neq 0$}

We consider now a big class of reducible representations consisting of the two irreducible components $S(m+1, \varrho)$ and $S(m-1, \varrho)$. The parameter $\varrho$ is arbitrary real, but should be taken equal zero if parity covariance is required. The reduced $\Gamma$-matrices $\beta$ are $2 \times 2$ matrices.

With the two differential operators $\Gamma_{0}$ defined in section III we obtain:

where

$$
\beta_{m+1, m-1}^{j}=+\left[\left(j+\frac{1}{2}\right)^{2}-j_{0}^{2}\right]^{\frac{1}{2}} \cdot\left(\begin{array}{ccc}
0 & e^{-i \varphi} \\
e^{i \varphi} & 0
\end{array}\right),
$$

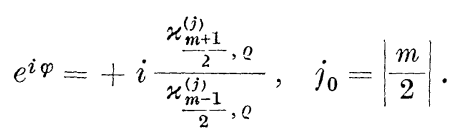

With the two differential operators $\Gamma_{3}$ applied to the pseudo-basis we get:

$$
\begin{aligned}
& \beta_{m+1, m-1}^{(\xi, \eta),+}=\left(\begin{array}{cc}
0 & \xi+i \frac{m}{2} \\
\xi-i \frac{m}{2} & 0
\end{array}\right), \\
& \beta_{m+1, m-1}^{(\xi, n),-,}=-\beta_{m,+, 1, m-1}^{(\xi, \eta),+}, \\
& \beta_{m+1, m-1}^{(k, \sigma)}=+\left[j_{0}^{2}-\left(k-\frac{1}{2}\right)^{2}\right]^{\frac{1}{2}}\left(\begin{array}{cc}
0+i \\
-i & 0
\end{array}\right), \\
& \beta_{m+1, m-1}^{(k,-\sigma)}=-\beta_{m+1, m-1}^{(k, \sigma)} .
\end{aligned}
$$

The mass formula can in all cases be written

where $J$ takes the values:

$$
M^{2}=\frac{\varkappa^{2}}{\left(J+\frac{1}{2}\right)^{2}-j_{0}^{2}}
$$

$J=j_{0}+$ positive half integer,

$J$ is twofold degenerate, there is a positive and a negative timelike solution, $J=-\frac{1}{2}+i \xi, 0<\xi<\infty$,

$J$ is fourfold degenerate, all eigenvalues $M^{2}$ are improper, $J=-j_{0}+$ positive half integer, $J \leqq-1$, 
$J$ is fourfold degenerate, there are two solutions of the positive and two solutions of the negative branch of the discrete series of $S U(1,1)$. The case $j_{0}=\frac{5}{2}$ is depicted in Fig. 2 .

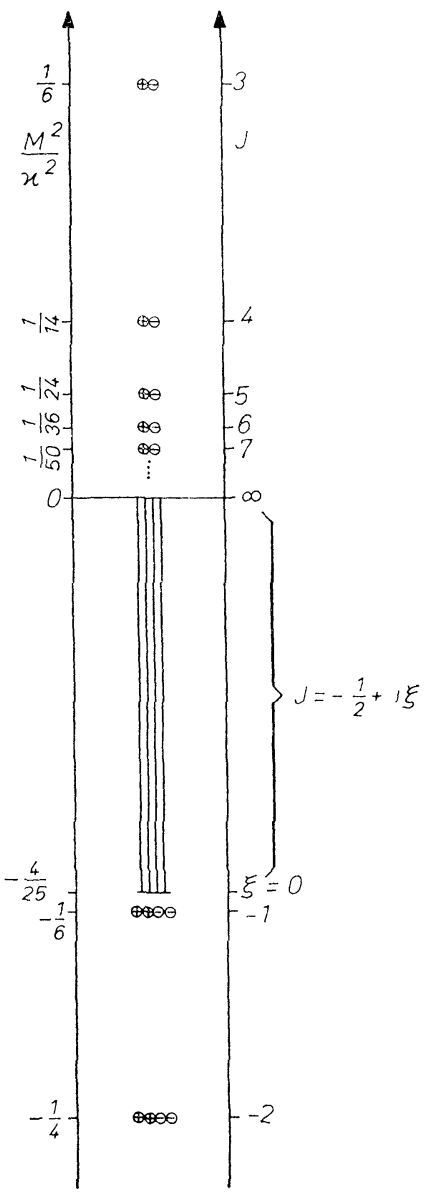

Fig. 2. $S_{1}=S(4, \varrho), S_{2}=S(6, \varrho)$

\section{Conclusion}

Representations with three or more irreducible components yield $M^{2}$-spectra which are superpositions of spectra of the type found above. They show the following typical properties:

a) The positive part is discrete and bounded from above with an accumulation point at $M^{2}=0$. 
3) The negative part consists always of a continuum which starts at $M^{2}=0$ and in some cases of an additional finite set of proper eigenvalues.

Acknowledgement. We thank Dr. J. Wess for the permission to use his unpublished results. We thank Dr. W. J. Holmax at the Duke University for calling our attention to the paper, Ref. [9]. It is a great pleasure to thank Professor A. PAIS for his kind hospitality at the Rockefeller University.

\section{Appendix: A Canonical Form for the Matrices which Represent Elements of $S \boldsymbol{U}(2)$ or $S U(1,1)$}

\section{The Principal Function}

We define a function

$$
Y_{q p}^{J}(z, \bar{z})=\frac{1}{2 \pi} \int_{0}^{2 \pi} e^{i(p-q) \vartheta}\left[1+z e^{-i \vartheta}\right]^{+p+J}\left[1+\bar{z} e^{i \vartheta}\right]^{-p+J} d \vartheta
$$

for any complex $J$ and parameters $p, q$ which are simultaneously integer or half integer. In the case where $p+J$ is integer, $z$ and $\bar{z}$ may be considered as independent variables. If $p+J$ is complex, $w^{p+J}$ has a cut from $w=0$ to $w=\infty$. If $\bar{z}$ is considered as the complex conjugate of $z, w^{p+J} \cdot \bar{w}^{-p+J}$ is a continuous function along the path of integration even for $|z|=|\bar{z}|>1$.

We call this function $I_{q p}^{J}$ the principal function, since many other functions can be regarded as special cases of it. We find the following properties:

$$
\begin{aligned}
& I_{q p}^{J}(z, \bar{z})=\overline{I_{-q,-p}(z, \bar{z})}, \\
& I_{q p}^{J}(z, \bar{z})=\bar{z}^{-p+J} z^{p+J} I_{q,-p}^{J}\left(\bar{z}^{-1}, z^{-1}\right) .
\end{aligned}
$$

It can be expanded into a series:

$p \geqq q,|z|,|\bar{z}|<1$ :

$p \leqq q,|z|,|\bar{z}|<1$ :

$$
I_{q p}^{J}(z, \bar{z})=\sum_{n=0}^{\infty}\left(\begin{array}{c}
-p+J \\
n
\end{array}\right)\left(\begin{array}{c}
+p+J \\
n+p-q
\end{array}\right) z^{n+p-q} \bar{z}^{n}
$$

$$
I_{q p}^{J}(z, \bar{z})=\sum_{n=0}^{\infty}\left(\begin{array}{c}
+p+J \\
n
\end{array}\right)\left(\begin{array}{c}
-p+J \\
n+q-p
\end{array}\right) z^{n} \bar{z}^{n+q-p}
$$

$p+q \geqq 0,|z|,|\bar{z}|>\mathrm{l}:$

$$
I_{q p}^{J}(z, \bar{z})=\sum_{n=0}^{\infty}\left(\begin{array}{c}
-p+J \\
n
\end{array}\right)\left(\begin{array}{c}
+p+J \\
p+q+n
\end{array}\right) z^{-n-q+J} \bar{z}^{-n-p+J},
$$

$p+q \leqq 0,|z|,|\bar{z}|>1:$

$$
I_{q p}^{J}(z, \bar{z})=\sum_{n=0}^{\infty}\left(\begin{array}{c}
+p+J \\
n
\end{array}\right)\left(\begin{array}{c}
-p+J \\
n-q-p
\end{array}\right) z^{-n+p+J} \bar{z}^{-n+q+J} .
$$


The principal function can also be expressed by Gaussian type hypergeometric functions:

$p \geqq q: \quad I_{q p}^{J}(z, \bar{z})=\left(\begin{array}{c}p+J \\ p-q\end{array}\right) \cdot z^{p-q}{ }_{2} F_{1}(-J+p,-J-q ; p-q+1 ; z \bar{z})$,

$p \leqq q: \quad I_{q p}^{J}(z, \bar{z})=\left(\begin{array}{c}-p+J \\ q-p\end{array}\right) \cdot \bar{z}^{q-p}{ }_{2} F_{1}(-J+q,-J-p ; q-p+1 ; z \bar{z})$.

\section{Wigner's Function $D_{q p}^{j}(u)$}

A unitary representation of the group $S U(2)$ to the spin $j$ can be realized in a space of polynomials of order $2 j$ of the variable $z$ by

$$
T_{u} f(z)=\left(u_{12} z+u_{22}\right)^{2 j} f\left(\frac{u_{11} z+u_{21}}{u_{12} z+u_{22}}\right) .
$$

In this space we take as a basis the functions

$$
f_{p}(z)=\frac{z^{j+p}}{\sqrt{(j+p) !(j-p) !}}, p=-j,-j+1, \ldots,+j .
$$

With the formal scalar product

we define

$$
\left(f_{q}, f_{p}\right)=\delta_{q p}
$$

$$
\begin{aligned}
D_{q p}^{j}(u)= & \left(f_{q}, T_{u} f_{p}\right) \\
= & {\left[\frac{(j+q) !(j-q) !}{(j+p) !(j-p) !}\right]^{\frac{1}{2}} \cdot \frac{1}{2 \pi} \int_{0}^{2 \pi} e^{i(p-q) \vartheta}\left[u_{21} e^{-i \vartheta}+u_{11}\right]^{j+p} \times } \\
& \times\left[u_{12} e^{+i \vartheta}+u_{22}\right]^{j-p} d \vartheta .
\end{aligned}
$$

We introduce the parameters

This yields

$$
\begin{aligned}
& u_{11}=+t^{\frac{1}{2}} \cdot e^{i \theta_{1}}, \quad u_{22}=\bar{u}_{11}, \\
& u_{12}=-(1-t)^{\frac{1}{2}} e^{i \theta_{2}}, \quad u_{21}=-\bar{u}_{12}, \\
& 0 \leqq t \leqq 1, \quad 0 \leqq \theta_{1}, \theta_{2}<2 \pi
\end{aligned}
$$

where

$$
D_{q p}^{j}(u)=[]^{\frac{1}{2}} \cdot e^{i \theta_{1}(q+p)+i \theta_{2}(q-p)} d_{q p}^{j}(t),
$$

$$
\begin{aligned}
d_{q p}^{j}(t)= & \frac{1}{2 \pi} \int_{0}^{2 \pi} e^{i(p-q) \vartheta}\left[t^{\frac{1}{2}}+(1-t)^{\frac{1}{2}} e^{-i \vartheta}\right]^{j+p} \times \\
& \times\left[t^{\frac{1}{2}}-(1-t)^{\frac{1}{2}} e^{+i \vartheta}\right]^{j-p} d \vartheta \\
= & t^{j} \cdot I_{q p}^{j}\left(\left[\frac{1-t}{t}\right]^{\frac{1}{2}},-\left[\frac{1-t}{t}\right]^{\frac{1}{2}}\right) .
\end{aligned}
$$

$j$ is integer or half integer, $j \pm p$ is integer, $z$ and $\bar{z}$ are regarded as independent variables. In terms of hypergeometric functions (poly- 
nomials) we get:

$$
\begin{aligned}
p \geqq q: \quad d_{q p}^{j}(t) & =t^{j-\frac{1}{2}(p-q)}(1-t)^{\frac{1}{2}(p-q)} \times \\
& \times\left(\begin{array}{l}
p+j \\
p-q
\end{array}\right) \cdot{ }_{2} F_{1}\left(-j+p,-j-q ; p-q+1 ;-\frac{1-t}{t}\right), \\
p \leqq q: \quad d_{q p}^{j}(t) & =(-1)^{q-p} t^{j-\frac{1}{2}(q-p)}(1-t)^{\frac{1}{2}(q-p)} \times \\
\times & \left(\begin{array}{c}
-p+j \\
q-p
\end{array}\right) \cdot{ }_{2} F_{1}\left(-j+q,-j-p ; q-p+1 ;-\frac{1-t}{t}\right) .
\end{aligned}
$$

We note that the phase normalization used here is identical with the Condon-Shortley convention. From the integral representation for $d_{q p}^{j}(t)$ we find immediately the important relations

$$
\begin{aligned}
d_{q p}^{j}(1-t) & =(-1)^{j-p} d_{-q, p}^{j}(t), \\
d_{q p}^{J}(t) & =(-1)^{q-p} d_{-q, p}^{j}(t) .
\end{aligned}
$$

The normalized invariant measure on the group $S U(2)$ is

$$
d \mu(u)=\frac{1}{4 \pi^{2}} d t d \theta_{1} d \theta_{2}
$$

\section{Bargmann's Function $C_{q p}^{\chi}(v)$ [7]}

In the case of the group $S U(1,1)$ we may proceed in the same fashion.

3.1. The continuous series $\chi=\chi(\xi, \eta), 0<\xi<\infty, \eta=0, \frac{1}{2}$.

We define a Hilbert space of square integrable functions $\mathscr{L}^{2}\left(\mathfrak{\aleph}_{1}\right)$ by

$$
\mathscr{L}^{2}\left(\aleph_{1}\right)=\left\{f(w), w=e^{i \varphi}, \int_{0}^{2 \pi}|\hat{f}(w)|^{2} d \varphi<\infty\right\} .
$$

In this space we realize a representation of the series $\chi(\xi, \eta)$ by

$$
T_{v} f(w)=\left(v_{12} w+v_{22}\right)^{i \xi+\eta-\frac{1}{2}}\left(\bar{v}_{12} w^{-1}+\bar{v}_{22}\right)^{i \xi-\eta-\frac{1}{2}} \cdot f\left(\frac{v_{11} w+v_{21}}{v_{12} w+v_{22}}\right) .
$$

We define a canonical basis in $\mathscr{L}^{2}\left(\aleph_{1}\right)$ by

For the element

$$
\begin{gathered}
f_{m}(w)=(2 \pi)^{-\frac{1}{2}} \cdot w^{m}, \\
-\infty<m<+\infty, m \text { integer } .
\end{gathered}
$$

we find

$$
v_{\varphi}=\left(\begin{array}{cc}
e^{i \frac{\varphi}{2}} & 0 \\
& 0 \\
0 & e^{-i} \frac{\varphi}{2}
\end{array}\right)
$$

$$
T_{v_{\varphi}} f_{m}=e^{i(m-\eta) \varphi} f_{m} .
$$

Therefore we introduce new labels by

$$
p=m-\eta
$$


and get

$$
C_{q p}^{(\xi, \eta)}(v)=\frac{1}{2 \pi} \int_{0}^{2 \pi} e^{i(p-q) \vartheta} \cdot\left[v_{21} e^{-i \vartheta}+v_{11}\right]^{p+J} \cdot\left[v_{12} e^{+i \vartheta}+v_{22}\right]^{-p+J} d \vartheta
$$

where $J=-\frac{1}{2}+i \xi$.

With the parameters

we get

$$
\begin{gathered}
v_{11}=s^{\frac{1}{2}} e^{i \theta_{1}}, \quad v_{22}=\bar{v}_{11}, \\
v_{12}=(s-1)^{\frac{1}{2}} e^{i \theta_{2}}, \quad v_{21}=\bar{v}_{12}, \\
1 \leqq s<\infty, \quad 0 \leqq \theta_{1}, \theta_{2}<2 \pi
\end{gathered}
$$

and

$$
C_{q p}^{(\xi, \eta)}(v)=e^{i \theta_{1}(q+p)+i \theta_{2}(q-p)} c_{q p}^{(\xi, \eta)}(s)
$$

$$
\begin{aligned}
c_{q p}^{(\xi, \eta)}(s)= & \frac{1}{2 \pi} \int_{0}^{2 \pi} e^{i(p-q) \vartheta}\left[(s-1)^{\frac{1}{2}} e^{-i \vartheta}+s^{\frac{1}{2}}\right]^{p+J} \times \\
& \times\left[(s-1)^{\frac{1}{2}} e^{+i \vartheta}+s^{\frac{1}{2}}\right]^{-p+J} d \vartheta \\
= & s^{J} \cdot I_{q p}^{J}\left(\left[\frac{s-1}{s}\right]^{\frac{1}{2}},\left[\frac{s-1}{s}\right]^{\frac{1}{2}}\right) .
\end{aligned}
$$

From this form we get the relation

$$
c_{q p}^{(\xi, \eta)}(s)=c_{-q,-p}^{(\xi, \eta)}(s) .
$$

3.2. The discrete series $\chi(k, \pm), k=\frac{1}{2}, 1, \frac{3}{2}, \ldots$.

Let us define the Hilbert space $\mathfrak{G}_{k}^{+}$by

$\mathfrak{G}_{k}^{+}= \begin{cases}f(w), & f \text { analytic for }|w|<1, \\ & f \text { continuous for }|w| \leqq 1,\end{cases}$

$$
\left.\|f\|^{2}=\frac{2 k-1}{\pi} \int_{|w|<1}\left(1-|w|^{2}\right)^{2 k-2}|f(w)|^{2} d w\right\} .
$$

We define the canonical basis by

$$
\begin{aligned}
f_{m}(w) & =N_{m} \cdot w^{m}, \quad m \geqq 0, \text { integer }, \\
N_{m} & =\left(\begin{array}{c}
2 k-1+m \\
m
\end{array}\right)^{\frac{1}{2}} .
\end{aligned}
$$

The representation $\chi(k,+)$ can be realized on this space by

so that

$$
T_{v} f(w)=\left(v_{12} w+v_{22}\right)^{-2 k} f\left(\frac{v_{11} w+v_{21}}{v_{12} w+v_{22}}\right)
$$

$$
T_{v_{\varphi}} f_{m}=e^{i(m+k) \varphi} f_{m}
$$


With the new label

we find

$$
p=m+k
$$

with

$$
C_{q p}^{(k,+)}(v)=\left[\frac{(k+p-1) !(q-k) !}{(k+q-1) !(p-k) !}\right]^{\frac{1}{2}} e^{i \theta_{1}(q+p)+i \theta_{2}(q-p)} \cdot c_{q p}^{(k,+)}(s)
$$

$$
\begin{aligned}
c_{q p}^{(k,+)}(s)= & \frac{1}{2 \pi} \int_{0}^{2 \pi} e^{i(p-q) \vartheta} \cdot\left[(s-1)^{\frac{1}{2}} e^{-i \vartheta}+s^{\frac{1}{2}}\right]^{p-k} \times \\
& \times\left[(s-1)^{\frac{1}{2}} e^{+i \vartheta}+s^{\frac{1}{2}}\right]^{-p-k} d \vartheta \\
= & s^{-k} I_{q p}^{-k}\left(\left[\frac{s-1}{s}\right]^{\frac{1}{2}},\left[\frac{s-1}{s}\right]^{\frac{1}{2}}\right) .
\end{aligned}
$$

The series $\chi(k,-)$ can be realized by putting

$$
\begin{aligned}
C_{q p}^{(k,-)}(v) & =\overline{C_{-q,-p}^{(k,+)}(v)}, \\
c_{q p}^{(k,-)}(s) & =\overline{c_{-q,-p}^{(k,+)}(s)} .
\end{aligned}
$$

The invariant measure on $S U(1,1)$ can be normalized to

$$
d \mu(v)=\frac{1}{4 \pi^{2}} d s d \theta_{1} d \theta_{2} .
$$

\section{The Generators of $S L(2, C)$ in the Canonical Basis}

The infinitesimal elements $\frac{1}{2} \sigma_{k}, \frac{i}{2} \sigma_{k}$ of $S L(2, C)$ are represented by operators $H_{k}, F_{k}$ such that

$$
\begin{gathered}
U_{a}=1+i \sum_{k} \varepsilon_{k} H_{k}+i \frac{\Sigma}{k} \eta_{k} F_{k}+0\left(\varepsilon^{2}, \eta^{2}\right), \\
\varepsilon_{k}, \eta_{k} \text { real. }
\end{gathered}
$$

In $\mathscr{L}^{2}(Z)$ they can be written as the following differential operator forms

$$
\begin{aligned}
H_{ \pm} & =H_{1} \pm i H_{2}, \quad F_{ \pm}=F_{1} \pm i F_{2}, \\
\lambda & =-\frac{m}{2}+\frac{i \varrho}{2}-1, \quad \mu=+\frac{m}{2}+\frac{i \varrho}{2}-1 \\
H_{+} & =-z^{2} \frac{\partial}{\partial z}-\frac{\partial}{\partial \bar{z}}+\lambda z, \\
H_{-} & =+\frac{\partial}{\partial z}+\bar{z}^{2} \frac{\partial}{\partial \bar{z}}-\mu \bar{z} \\
H_{3} & =+z \frac{\partial}{\partial z}-\bar{z} \frac{\partial}{\partial \bar{z}}-\frac{1}{2}(\lambda-\mu) \\
F_{+} & =-i z^{2} \frac{\partial}{\partial z}+i \frac{\partial}{\partial \bar{z}}+i \lambda z, \\
F_{-} & =+i \frac{\partial}{\partial z}-i \bar{z}^{2} \frac{\partial}{\partial \bar{z}}+i \mu \bar{z} \\
F_{3} & =+i z \frac{\partial}{\partial z}+i \bar{z} \frac{\partial}{\partial \bar{z}}-\frac{i}{2}(\lambda+\mu) .
\end{aligned}
$$


$H_{ \pm}$and $H_{3}$ generate $S U(2), F_{ \pm}$and $H_{3}$ generate $S U(1,1)$. The canonical basis $\left\{f_{p}^{j}\right\}$ satisfies

$$
\begin{aligned}
& H_{3} f_{p}^{j}=p f_{p}^{j}, \\
& H_{+} f_{p}^{j}=+[(j-p)(j+p+1)]^{\frac{1}{2}} f_{p+1}^{j}, \\
& F_{+} f_{j}^{j}=+C_{j+1} \cdot[(2 j+1)(2 j+2)]^{\frac{1}{2}} f_{j+1}^{j+1},
\end{aligned}
$$

where $C_{j+1}$ is (see Ref. [5], page 117)

$$
C_{j}=\frac{i}{j}\left[\frac{\left(j^{2}-j_{0}^{2}\right)\left(j^{2}+\frac{\varrho^{2}}{4}\right)}{4 j^{2}-1}\right]^{\frac{1}{2}} .
$$

For the other matrix elements of the generators in the canonical basis we refer to the same source, Ref. [5], page 117. In our phase convention we have to insert, however,

$$
A_{j}=\frac{1}{4} \cdot \frac{\varrho \cdot m}{j(j+1)} .
$$

\section{References}

1. Majorana, E.: Nuovo Cimento 9, 335 (1932).

2. Gelfand, I. M., i A. M. YAGLom: Zh. Eksperim. i Teor. Fiz. 18, 703, 1096 (1948).

3. Bargmann, V.: Math. Rev. 10, 583, 584 (1949).

4. Rideau, G.: Comm. Math. Phys. 3, 218 (1966).

5. NAImark, M. A.: Linear representations of the Lorentz group. Oxford: Pergamon Press 1964.

6. - Normed rings. $\S 29$. Groningen: Noordhoff 1959 .

7. Bargmann, V. A.: Ann. Math. 48, 568 (1947).

8. Harish-Chandra: Proc. Nat. Acad. Sci. U.S. 38, 337 (1952).

9. Sciarrino, A., and M. Toller: Università di Roma, Nota interna, October 1966.

10. Naimark, M. A.: Am. Math. Soc. Transl., Series 2 36, 101 (1964).

11. RüHL, W.: Nuovo Cimento 44, 659 (1966).

12. Ryzhiк, I. M., and I. S. Gradshteyn: Tables of integrals, series and products. New York: Academic Press 1965. 